\title{
O Centro de Apoio Educacional e Psicológico (CAEP) da Faculdade de Medicina de Ribei- rão Preto (USP): História e perspectivas
}

\begin{abstract}
Ana Raquel L. Cianflone ${ }^{1}$; José Fernando C. Figueiredo ${ }^{2}$ \& Maria de Fátima A. Colares ${ }^{3}$
${ }^{1}$ Docente. Departamento de Psicologia e Educação. Faculdade de Filosofia, Ciências e Letras de Ribeirão Preto - USP. Pedagoga do CAEP da FMRP-USP de 1989 a 2001. ²Docente. Departamento de Clínica Médica. Faculdade de Medicina de Ribeirão Preto - USP. Coordenador do CAEP de 1997 a 2001. ${ }^{3}$ Psicóloga do Centro de Apoio Educacional e Psicológico da FMRP-USP.

Correspondencia: Avenida Bandeirantes, 3900. CEP: 14040-901.Ribeirão Preto - SP. E-mail: arlcianflone@ffclrp.usp.br
\end{abstract}

\section{A CRIAÇÃO}

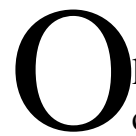
Centro de Apoio Educacional e Psicológico (CAEP) da Faculdade de Medicina de Ribeirão Preto -USP, inicialmente denominado Núcleo de Apoio Psicopedagógico (NAPP), foi implantado no primeiro semestre de 1990. Apesar de ter sido fundado na década de 90, vários documentos internos - manifestações individuais de professores, de departamentos e pareceres de colegiados - já registravam, desde a década de 60 , a preocupação com alunos considerados emocionalmente comprometidos, o que, a princípio, estaria prejudicando seu desenvolvimento profissional.

Nesse contexto institucional, a Comissão de Graduação, especialmente no período em que foi presidida pelo Prof.Dr. Cláudio Roberto Carvalho Rodrigues, docente do Departamento de Psiquiatria, foi o fórum principal de discussão sobre a necessidade de atenção às dificuldades emocionais e acadêmicas, vivenciadas pelos alunos ao longo do curso. Certamente, a formação acadêmica e a experiência clínica do então presidente da Comissão de Graduação com problemas dessa ordem e o seu grande envolvimento com a formação do médico em nível de graduação, fertilizaram as discussões no âmbito da instituição sobre as questões relacionadas à saúde mental do estudante de Medicina. As discussões iniciais para a elaboração do projeto de implantação do CAEP evidenciaram que a expectativa principal com a criação de um serviço de apoio psicológico era de que este, de alguma forma, se responsabilizasse pela assistência psicológica ao estudante e, especialmente, que resolvesse situações de impasse, como, por exemplo, os casos de alunos com quadros psicóticos graves ou transtornos de conduta. Apesar de raros, esses casos, quando ocorriam, causavam grande preocupação em toda a comunidade acadêmica. No entanto, no transcorrer das discussões, houve um nítido amadurecimento dessa proposta inicial e um ajustamento das expectativas iniciais às reais possibilidades de atuação de um serviço dessa natureza. Assim, predominou a compreensão de que, em casos como os acima mencionados, o CAEP teria um papel importante a desempenhar, porém não exclusivo, pois a responsabilidade pela assistência ao aluno seria de toda a instituição e não apenas de um único setor.

Assim, foi definido o primeiro princípio básico da concepção do projeto de criação do CAEP: a atuação deveria se voltar não apenas para o aluno e seus eventuais problemas, mas a todo o campo institucional. Embora muitos problemas e dificuldades se manifestem nos estudantes, estes se situam num contexto institucional no qual estão inseridos também professores e funcionários e que possui um modo próprio de funcionamento. Desse princípio norteador, derivou 
todo o processo de estruturação do centro, que foi transposto, posteriormente, para o seu Regulamento Interno. A adoção desse princípio não implicou no prejuízo do caráter assistencial do CAEP, mas, sim, procurou ampliar seu campo de intervenção, contribuindo, assim, de modo mais amplo, para a melhoria de todo o processo de formação do médico.

Ao considerar que a atuação do CAEP deveria envolver todo o campo institucional, sem perder de vista o caráter assistencial que motivou originalmente a sua criação, estabeleceu-se uma situação bastante complexa e desafiadora. Ao mesmo tempo em que era proposta uma atuação no campo institucional, era também necessária a criação de um setting adequado para o trabalho de assistência ao estudante. Para isso, era primordial o estabelecimento de um vínculo de confiança entre o CAEP e o aluno, o que envolvia, necessariamente, um certo distanciamento da dinâmica institucional, ou seja, era essencial que o CAEP fosse considerado pelos alunos como um espaço exclusivo e confiável para a solicitação de ajuda, mesmo quando a fonte de suas dificuldades fosse a própria instituição. Assim, ao longo de sua história, o CAEP vem atuando, metaforicamente, em um movimento pendular, estando ora dentro, ora fora da instituição. Consideramos que, no transcorrer dos 12 anos de existência, a despeito das muitas dificuldades enfrentadas, foi possível encontrar muito mais complementaridade entre essas duas vertentes de atuação do que divergências. Entendemos que o crescimento da procura espontânea dos alunos por atendimento, associado a outros indicadores diretos e indiretos, reflete que, de fato, foi possível a criação de um espaço institucional específico para o estudante. Certamente o CAEP hoje se configura como um local legítimo de referência para os alunos, tanto para assistência, como para orientação e encaminhamentos. Nos tópicos seguintes, explicitaremos como vêm se desenvolvendo essas duas vertentes.

\section{PRINCÍPIOS DE FUNCIONAMENTO, GES- TÃO E COMPOSIÇÃO DA EQUIPE}

Desde as discussões iniciais, que levaram à elaboração do projeto de implantação do CAEP, estabeleceu-se que, dentre as atividades essenciais, deveriam estar o desenvolvimento de estudos sistemáticos, que levassem a conhecer e compreender as características e necessidades da população discente, como ponto de partida para o desenvolvimento de medidas de intervenção. Com base nesse princípio, foram definidos os objetivos principais do CAEP em seu Regulamento Interno:

"Artigo $1^{\circ}$ - As atividades do CAEP destinamse a todos estudantes do curso de graduação da FMRP e tem como objetivos:

A. O desenvolvimento de estudos e projetos em Educação Médica, com a finalidade de oferecer subsídios para o aprimoramento do ensino nesta Unidade.

B. O desenvolvimento de atividades de suporte psicopedagógico e psicológico aos alunos do curso de graduação, com ênfase na identificação das características acadêmicas, emocionais e na prevenção de dificuldades de aprendizagem e/ou psicológicas.

C. O desenvolvimento de estudos e projetos com o objetivo de obter a caracterização pedagógica, psicossocial, e psicopedagógica da população discente.

D. Prestar assessoria à Comissão de Graduação e aos docentes da FMRP, no âmbito de suas atribuições".

Desde sua criação, o CAEP possui uma equipe técnica, composta por uma psicóloga, uma pedagoga e um técnico acadêmico para apoio administrativo. A gestão e a administração do CAEP estão divididas em instâncias distintas. A primeira, denominada Grupo de Consultores, é constituída por membros do corpo docente e por um representante discente. Os membros docentes são indicados pela Comissão de Graduação e o membro discente é indicado pelo representante discente na Comissão de Graduação. O Grupo de Consultores foi criado no ano de 1992, por solicitação da própria equipe técnica do CAEP à Comissão de Graduação. Tal solicitação visava estabelecer maior nível de interlocução entre a equipe técnica e os demais membros da instituição para definir as principais diretrizes do Centro de Apoio Educacional e Psicológico e, também, para refletir sobre situações complexas, que exigissem análises específicas. O Grupo de Consultores assessora tanto a equipe técnica quanto a Comissão de Graduação, à qual o centro é subordinado, nas questões relativas ao CAEP.

Além do Grupo de Consultores, há também um Coordenador, indicado pela Comissão de Graduação, que tem como função: traçar diretrizes, supervisionar as atividades desenvolvidas e deliberar sobre solicitações encaminhadas ao CAEP. O Coordenador tam- 
bém é responsável pela convocação do Grupo de Consultores e pela coordenação das reuniões. Todos os mandatos, de Coordenador e dos membros do Grupo de Consultores, são de dois anos, sendo permitidas reconduções. A última instância de administração e gestão do CAEP é a Comissão de Graduação da FMRP-USP.

É importante destacar que, regimentalmente, é vedada aos membros da equipe técnica a participação em comissões de sindicância, atividades periciais e elaboração de laudos psicológicos. Além dessas restrições estabelecidas em regimento, a equipe técnica zela rigorosamente pelo não envolvimento com quaisquer situações que envolvam medidas disciplinares da instituição com os alunos.

Cabe mencionar, também, o incentivo e o apoio da instituição à qualificação permanente dos membros da equipe técnica, que vêm desenvolvendo, ao longo dos anos, projetos de pesquisa estreitamente relacionados à área de atuação do CAEP. Tais projetos, além de terem sido desenvolvidos a partir de questões formuladas no cotidiano do trabalho, também trouxeram importantes subsídios para o aprimoramento da atuação do centro em diferentes níveis.

Considerando o que foi exposto anteriormente, é possível notar que o trabalho de investigação de características e necessidades do estudante de Medicina, assim como as peculiaridades de sua formação, constituíram o fundamento e o ponto de partida para a proposição de todas as medidas de intervenção do CAEP.

Nessa perspectiva, vários trabalhos foram realizados e posteriormente divulgados na revista Medicina, Ribeirão Preto, com o objetivo de devolver, num primeiro momento, aos alunos e professores da FMRPUSP os resultados de estudos dos quais eles tivessem participado e, também, de propiciar uma visão peculiar e complementar da realidade por eles vivenciada. As divulgações também tinham como objetivo oferecer subsídios para as discussões sobre educação médica no âmbito da instituição e assim ampliar o campo de atuação do CAEP.

Dentre os trabalhos realizados, vale destacar alguns que tiveram grande importância para a elaboração de diretrizes do CAEP e que também proporcionaram uma melhor compreensão da realidade institucional e do processo de formação do médico. A primeira divulgação elaborada pela equipe técnica foi o próprio projeto inicial de implantação do serviço, intitulado "O Núcleo de Apoio Educacional e Psi- cológico da Faculdade de Medicina de Ribeirão Preto - USP" (1). Tal publicação teve como principal objetivo divulgar amplamente as diretrizes iniciais do centro, possibilitando, assim, maior envolvimento e participação da comunidade acadêmica. $\mathrm{O}$ primeiro trabalho de pesquisa propriamente dito, originário do CAEP, foi o "Estudo retrospectivo de uma população de alunos de Medicina atendidos no ambulatório de clínica psiquiátrica do Hospital das Clínicas da Faculdade de Medicina de Ribeirão Preto"(2), realizado pela psicóloga do CAEP e pelo coordenador do ambulatório de psiquiatria do Hospital das Clínicas. Esse estudo teve como objetivo conhecer o perfil da demanda de estudantes de graduação que procuravam por assistência no ambulatório de Psiquiatria, pois, até 1990, era a única possibilidade de assistência à saúde mental oferecida pela instituição. Esse trabalho foi de fundamental importância para a proposição das diferentes modalidades de assistência ao aluno, pois permitiu um conhecimento mais aprofundado da população a ser atendida.

Posteriormente, foram realizados outros estudos, intitulados "Algumas características do ensino e aprendizado na Faculdade de Medicina de Ribeirão Preto - USP" (3) e "Contribuição de diferentes atividades para o processo de aprendizagem na percepção de estudantes de Medicina” (4), que tiveram como objetivo conhecer melhor como os estudantes vivenciavam o processo de ensino-aprendizado e as possíveis implicações dessas vivências para a formação e desenvolvimento profissional do médico.

A escolha da profissão médica também foi objeto de investigação no estudo "A representação da Medicina como profissão: considerações teóricas sobre conteúdos levantados junto a estudantes secundaristas e universitários"(5). Essa pesquisa foi realizada junto a vestibulandos para o curso de Medicina e estudantes dos primeiros três anos do curso médico. A motivação inicial para a proposição do estudo foi a alta incidência de intenção de abandono do curso, registrada nos atendimentos realizados pelo CAEP. Apesar dos índices de evasão e abandono serem baixos, a manifestação dessa intenção e as insatisfações apontadas pelos alunos revelaram a necessidade de grandes esforços adaptativos, que, via de regra, resultam em sofrimento e problemas acadêmicos para os estudantes.

Ainda no âmbito da formação médica, mais especificamente em relação ao ciclo básico, o estudo 
intitulado: "As fontes de tensão no curso médico: um estudo psicométrico" (6) possibilitou a identificação de tendências atitudinais dos estudantes de Medicina frente ao ciclo básico, apontando conflitos nesse período da formação. $\mathrm{O}$ estudo contribuiu, portanto, para a identificação precoce dessas dificuldades, podendo auxiliar no estabelecimento de diretrizes para intervenções psicológicas, que priorizassem ações preventivas e de promoção de saúde mental dos estudantes. Além disso, os resultados obtidos oferecem subsídios para a elaboração de estratégias educacionais, que possam contribuir para a formação de atitudes positivas dos estudantes frente ao estresse no curso médico.

Merece destaque, ainda, a atuação do CAEP no Grupo de Trabalho constituído pela Comissão de Graduação para a avaliação terminal de competências dos graduandos da Faculdade de Medicina, levada a efeito no período de 1993 a 2.000. Nesse processo, o CAEP atuou diretamente, auxiliando e dando subsídios técnicos durante a elaboração das diferentes formas de avaliação, suas aplicações, correções e análise dos resultados.

\section{MODALIDADES DE ASSISTENCIA E CAMPO DE ATUAÇÃO}

O CAEP tem como público-alvo para assistência psicológica e psicopedagógica os alunos de graduação da FMRP. O objetivo geral principal desse trabalho é auxiliar o estudante no seu desenvolvimento pessoal e profissional, no decorrer de sua formação acadêmica. As modalidades de atendimento são variáveis e procuram se adaptar à disponibilidade dos alunos e às possibilidades do serviço. De modo geral, as modalidades de assistência são as mencionadas a seguir.

Psicoterapia breve - Após algumas entrevistas de avaliação, se houver indicação, realiza-se o atendimento psicoterápico, geralmente, com sessões semanais individuais. Quando há necessidade, o número de sessões pode ser maior.

Orientação psicopedagógica - Esse trabalho visa desenvolver, nos estudantes, recursos adaptativos, especialmente os metacognitivos, que implicam na construção de uma nova postura de estudante; busca a aquisição de novos hábitos de estudo e novas formas de conduzir o próprio aprendizado ao longo da vida. Consideramos que esse trabalho tem uma importante característica preventiva, pois permite evitar sentimentos de baixa estima e desvalia, oriundos de um desempenho acadêmico insatisfatório. Essa é uma intercorrência comum na formação médica, pois é freqüente estudantes, considerados brilhantes durante o ensino médio e com uma história escolar de sucesso, experimentarem dificuldades na vida acadêmica e desempenhos insatisfatórios nas avaliações, especialmente no início do curso.

Orientação profissional - Tem como objetivo contribuir na preparação do aluno para o enfrentamento de eventuais dificuldades relacionadas ao processo de formação profissional e/ou ao próprio exercício da profissão médica. Visa também, quando necessário, reorientar o estudante no processo de escolha de uma outra profissão. Também pode ser realizado um trabalho direcionado para a escolha da especialidade com estudantes das etapas finais da graduação.

Grupos temáticos e de reflexão - São grupos que podem ser formados a partir de temas propostos pelos próprios estudantes, geralmente relacionados ao desenvolvimento da identidade profissional e ao papel do médico. Os grupos reflexivos representam uma estratégia profilática, que permite aos estudantes vivenciarem e compartilharem, em grupo, suas angústias, medos e incertezas, em torno de situações que são comuns a todos, podendo levar a reflexões mais positivas e críticas frente ao papel profissional, que está em desenvolvimento.

Orientação familiar - Tem como objetivo orientar a conduta de familiares de alunos em caso de necessidade. Sempre é feita com conhecimento e anuência do estudante envolvido.

Orientação a professores - Esse trabalho visa oferecer subsídios para que o docente, de alguma forma, favoreça a superação de dificuldades psicológicas, enfrentadas por seus alunos. Essa orientação é feita a partir da iniciativa do próprio professor e, caso o aluno esteja em atendimento no CAEP, a orientação só ocorre com o conhecimento e anuência dele.

Orientação e encaminhamentos - São realizados especialmente em situações que exijam procedimentos de competência medico-psiquiátrica, não realizados pelo CAEP, ou que necessitem de um processo psicoterápico prolongado.

Assessoria pedagógica a docentes - Procura oferecer ao docente subsídios originários das ciências da educação para o aprimoramento de sua prática pedagógica.

Assessoria pedagógica à Comissão de Graduação - São ações de colaboração, no âmbito das 
atribuições do CAEP, com diferentes atividades desenvolvidas por esse colegiado que envolvam o ensino de graduação.

É importante ressaltar que, ao longo da existência do CAEP, a observância dos princípios estabelecidos desde o início, a coesão e estabilidade da equipe técnica, dos consultores e da coordenação, associados ao estabelecimento de um ambiente acolhedor e confiável para os estudantes, contribuíram substancialmente para sua solidificação e para o estabelecimento de novas metas.

\section{PERSPECTIVAS}

Neste momento, o CAEP, em conjunto com seu Grupo de Consultores, elabora o projeto de implantação de Programa de Tutoria, na instituição. Esse programa tem, inicialmente, dentre seus principais objetivos, trabalhar as motivações do aluno para a profissão, favorecendo discussões sobre a vida acadêmica do aluno, de modo a proporcionar suporte e facilitar o caminho para as ações do futuro profissional médico. De forma gradativa, o mesmo programa poderá estender-se aos cursos novos, tendo em vista a integração entre eles, e a implementação de discussões a respeito do trabalho multiprofissional, acompanhando, assim, o novo momento da Faculdade de Medicina com a implantação de outros cursos na área da saúde.

O Centro de Apoio Educacional e Psicológico, portanto, tem procurado desempenhar um papel que inclui não apenas a assistência aos estudantes, mas, também, o desenvolvimento de estudos e projetos que envolvam todo o campo institucional e as questões relevantes da formação médica. Dessa forma, e nessa mesma vertente, é que se pretende estabelecer diretrizes semelhantes de atuação em relação aos cursos novos da unidade. Assim, entendemos que atentar para a saúde mental do estudante universitário é tarefa muito ampla, que não compete exclusivamente aos profissionais de saúde mental, e sim, à instituição como um todo.

Nestes 12 anos de existência, o CAEP cresceu e se estabilizou como referência para os alunos, passando a ser reconhecido, também,fora do âmbito restrito da FMRP-USP. No entanto, o papel e o potencial do CAEP permanecem pouco conhecidos por grande parte do corpo docente da instituição, que ainda o vê como mero prestador de assistência psicológica aos alunos. A maior abrangência das atividades do CAEP, voltadas também para aspectos educacionais e peda- gógicos, fazem do centro o fórum ideal para a discussão de temas relacionados com o planejamento e a implementação de políticas educacionais na FMRPUSP, sob a tutela e incentivo da Comissão de Graduação. Com tais atividades, o CAEP, como centro gerador de conhecimentos aplicáveis na área educacional, pode vir a desempenhar, também, papel relevante no momento em que se estruturam os novos cursos na Faculdade de Medicina de Ribeirão Preto.

\section{REFERÊNCIAS BIBLIOGRÁFICAS}

1 - FERNANDES JM \& CIANFLONE ARL. O Núcleo de Apoio Psicopedagógico da Faculdade de Medicina de Ribeirão Preto - USP. Medicina, Ribeirão Preto, 24: 112-127, 1991.

2 - FERNANDEZ JM \& RODRIGUES CRC. Estudo retrospectivo de uma população de estudantes de medicina atendidos no ambulatório de clínica psiquiátrica do Hospital das Clínicas da Faculdade de Medicina de Ribeirão Preto. Medicina, Ribeirão Preto, 26: 258-269, 1993.

3 - CIANFLONE ARL \& FERNANDES JM. Algumas características do ensino e aprendizado na Faculdade de Medicina de Ribeirão Preto - USP - um estudo junto aos alunos de graduação. Medicina, Ribeirão Preto, 26: 228-236, 1993.

4 - FIGUEIREDO JFC; RODRIGUES MLV; CIANFLONE ARL \& COLARES MFA. Contribuição de diferentes atividades para o processo de aprendizagem, na percepção de alunos de Medicina. Medicina, Ribeirão Preto, 29: 383-388, 1996.

5 - CIANFLONE ARL \& FIGUEIREDO MAC. A representação da medicina como profissão: considerações teóricas sobre conteúdos levantados junto a estudantes secundaristas e universitários. Medicina, Ribeirão Preto, 26: 237-245, 1993.

6 - COLARES MFA As fontes de tensão no curso médico: um estudo psicométrico. Dissertação de Mestrado, Escola de Enfermagem de Ribeirão Preto da USP. Ribeirão Preto. p. $1-138,1999$ 